\title{
Suplementação excessiva com ácido fólico durante a gravidez e desenvolvimento de asma na infância: uma revisão baseada na evidência
}

Andreia Ramôa, ${ }^{1}$ Pedro Costa, ${ }^{2}$ Ana Maria Ribeiro, ${ }^{3}$ Stephanie De Castro ${ }^{4}$

\section{RESUMO}

Objetivo: A suplementação materna com ácido fólico tem um efeito positivo na prevenção de defeitos do tubo neural. É controversa a relação entre a suplementação com altas doses de ácido fólico $(5 \mathrm{mg})$ e a asma na criança. O objetivo desta revisão é conhecer a evidência da associação entre a suplementação com altas doses de ácido fólico durante a gravidez e o risco de desenvolvimento de asma na infância.

Fontes de dados: National Guideline Clearinghouse, Canadian Medical Association Pratice Guidelines, Cochrane Library, DARE, Bandolier, PubMed e Índex de Revistas Médicas Portuguesas.

Métodos de revisão: Pesquisa de meta-análises, revisões sistemáticas, estudos originais e normas de orientação clínica publicados entre julho/2006 e julho/2016 nas línguas portuguesa, inglesa, espanhola e francesa, utilizando os termos MeSH: Folic Acid, Asthma e Pregnancy. Foi utilizada a escala SORT (Strength of Recommendation Taxonomy), da American Academy of Family Physicians, para atribuição do nível de evidência e forças de recomendação.

Resultados: Foram selecionados cinco estudos de um total de 29 artigos encontrados: duas meta-análises e três estudos originais. As meta-análises não sustentam a associação entre a suplementação periconcecional com ácido fólico e o aumento do risco do desenvolvimento de asma na infância. No entanto, nenhum dos estudos incluídos nas meta-análises estratificam os resultados de acordo com a dose de ácido fólico fornecida à gestante durante a gestação e o risco de asma. Por outro lado, os estudos originais sugerem que a suplementação com altas doses de ácido fólico durante a gravidez pode aumentar o risco de desenvolvimento de asma na infância.

Conclusões: A evidência da associação entre a suplementação com ácido fólico durante a gravidez e o risco de desenvolvimento de asma durante a infância é controversa. Os estudos com resultados estratificados por dose de ácido fólico mostraram que altas doses de suplementação (>800 $\mathrm{\mu g}$ ) estão associadas ao aumento do risco de desenvolvimento de asma na infância (SORT B).

Palavras-chave: Ácido fólico; Asma; Gravidez.

\section{INTRODUÇÃO}

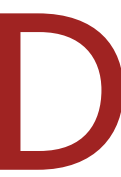
esde 1991 que são conhecidos os efeitos benéficos da suplementação com ácido fólico no período periconcecional e no primeiro trimestre de gravidez pela redução, até $78 \%,{ }^{1-4}$ do risco de defeitos do tubo neural e de outras anomalias congénitas. Neste sentido, a Organização Mundial da Saúde e a Direção-Geral da Saúde recomendam a suplementação diária com $400 \mu \mathrm{g}$ de ácido fólico nas mu- lheres sem fatores de risco durante este período. ${ }^{5-6}$ Está recomendada a suplementação com $5 \mathrm{mg}$ de ácido fólico em mulheres com risco acrescido de ter um filho com defeitos do tubo neural (filho anterior com defei-

1. USF Gualtar, ACeS Cávado I - Braga

2. USF Maxisaúde, ACeS Cávado I - Braga

3. USF São João de Braga, ACES Cávado I - Braga

4. USF + Carandá, ACES Cávado I - Braga 
to do tubo neural, história familiar desta situação, mulheres sob terapêutica associada à diminuição da biodisponibilidade de ácido fólico ou com outras doenças crónicas). ${ }^{7-9}$

A asma é uma doença respiratória crónica comum, com importante impacto na qualidade de vida das pessoas e cuja incidência tem vindo a aumentar. ${ }^{10}$

Alguns estudos têm mostrado uma associação entre o desenvolvimento de asma na infância e a suplementação materna com ácido fólico durante a gravidez. ${ }^{11-12}$ Conhecem-se os efeitos depressores de altas doses de folatos em circulação na citotoxicidade das células $\mathrm{Na}$ tural Killer e o papel dos folatos como dadores de grupos metilo que podem induzir alterações epigenéticas. Com base nestas características tem-se colocado em hipótese o aumento do risco de desenvolvimento de doenças alérgicas, nomeadamente a asma. ${ }^{13-14}$ Efetivamente, estudos experimentais em ratinhos e observacionais em humanos têm reforçado esse possível efeito adverso da suplementação com ácido fólico durante a gravidez. ${ }^{11-12,15}$

Crianças cujas mães são suplementadas durante a gravidez com uma dose de ácido fólico dez vezes superior ao recomendado, poderão estar desnecessariamente em risco de ocorrência de efeitos adversos, nomeadamente desenvolvimento de asma durante a infância. ${ }^{16}$

Em Portugal existe apenas uma formulação isolada de ácido fólico com 400ug. As restantes formulações contêm $5 \mathrm{mg}$. De referir ainda que a formulação de $400 \mu \mathrm{g}$ é a única formulação de ácido fólico isolado não comparticipada. Assim, estima-se que a maioria das gestantes, sem fatores de risco e sem indicação para suplementação com multivitamínicos, estará suplementada com ácido fólico numa dose muito superior à recomendada. ${ }^{17}$

Esta revisão tem como objetivo conhecer a evidência existente acerca da associação entre a suplementação materna com altas doses de ácido fólico durante a gravidez de baixo risco e o risco de desenvolvimento de asma na infância.

\section{MÉTODOS}

Foi realizada uma pesquisa bibliográfica em agosto de 2016, por dois autores da revisão, utilizando os termos MeSH Folic Acid, Asthma e Pregnancy, que fo- ram concordantes entre si. Foi selecionada a pesquisa de meta-análises (MA), revisões sistemáticas (RS), estudos originais (EO) e normas de orientação clínica (NOC) nas bases de dados da National Guideline Clearinghouse, NICE, Canadian Medical Association Practice Guidelines, Cochrane, DARE, Bandolier, PubMed e Índex de Revistas Médicas Portuguesas dos artigos publicados entre julho de $2006 \mathrm{e}$ julho de 2016 nas línguas portuguesa, inglesa, espanhola e francesa.

A população em estudo incluiu mulheres grávidas ou crianças até aos seis anos de idade, cuja suplementação materna com ácido fólico durante a gestação fosse conhecida. A intervenção avaliada foi a suplementação excessiva com ácido fólico durante a gravidez (5mg) em comparação com a suplementação com as doses recomendadas $(400 \mu \mathrm{g})$. O outcome avaliado foi a incidência de asma durante a infância. Foram excluídos os estudos que incluíam grávidas em risco de ter um filho com defeitos do tubo neural (i.e., com filho anterior com defeito do tubo neural, história familiar desta situação, mulheres sob terapêutica associada à diminuição da biodisponibilidade de ácido fólico ou com outras doenças crónicas), estudos que tinham outros outcomes, artigos duplicados, artigos de opinião, artigos de revisão clássica e artigos discordantes com o objetivo da revisão.

Foi utilizada a escala SORT (Strength of Recommendation Taxonomy), da American Academy of Family Physicians, para atribuição dos níveis de evidência (NE) e forças de recomendação (FR). ${ }^{18}$

\section{RESULTADOS}

Da pesquisa foram obtidos 29 artigos, dos quais foram selecionados quatro (o que correspondeu a cinco estudos, uma vez que um dos artigos incluiu dois estudos - uma meta análise e um estudo original). O processo de seleção dos artigos incluídos está representado na Figura 1.

A descrição resumida dos artigos incluídos nesta revisão encontra-se no Quadro I.

A MA de Crider e colaboradores, ${ }^{19} \mathrm{de} 2013$, teve como objetivo principal verificar a existência de associação entre a suplementação com ácido fólico durante a gravidez e o risco de desenvolvimento de asma e outras doenças alérgicas durante a infância. Esta MA incluiu 


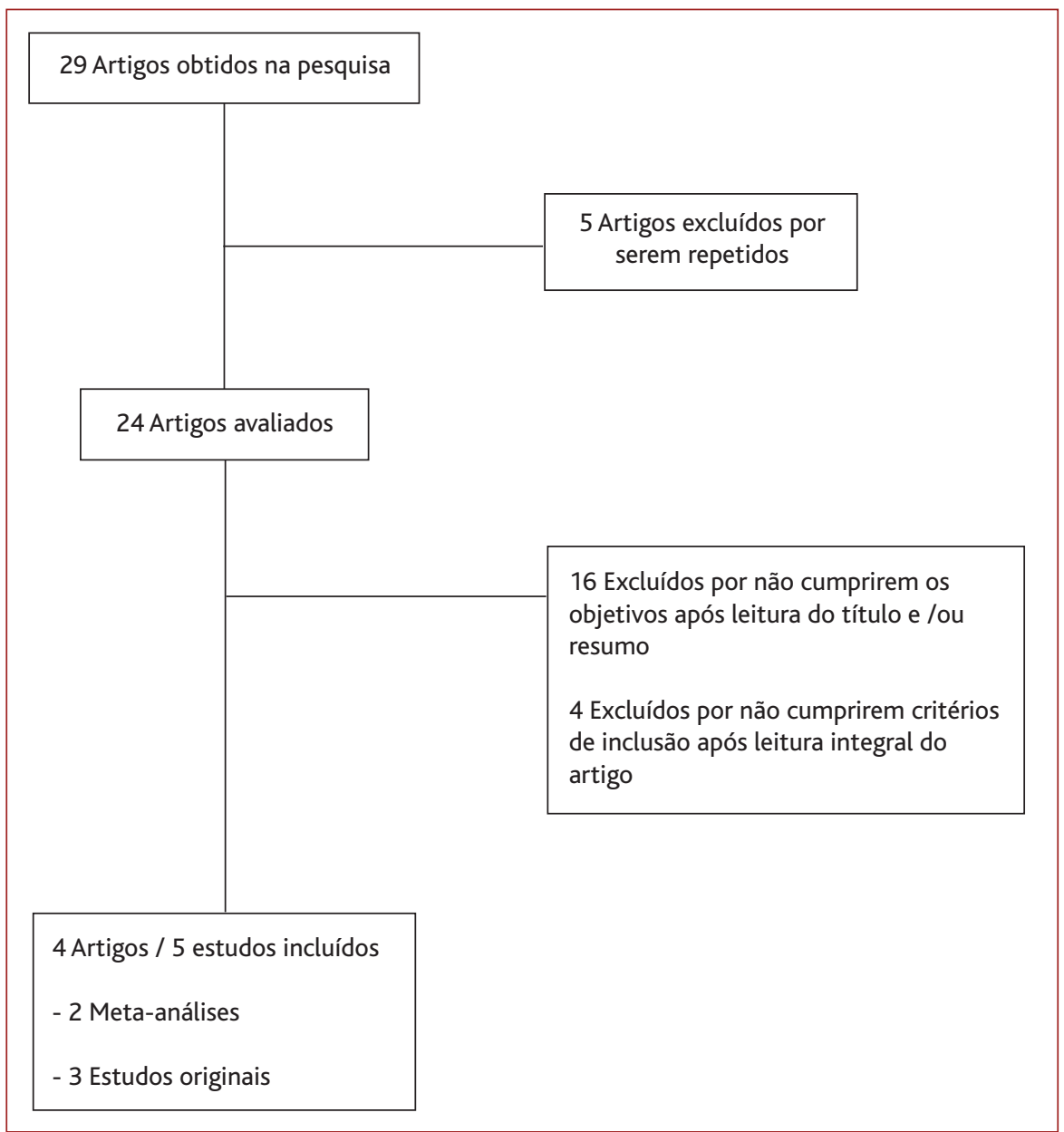

Figura 1. Diagrama de avaliação dos artigos obtidos.

cinco estudos (três estudos coorte e dois estudos caso-controlo). Foram analisados os efeitos da suplementação com ácido fólico no período periconcecional e primeiro trimestre, comparando com a não suplementação, no que diz respeito ao desenvolvimento de asma ou pieira na infância. Na totalidade foram estudadas 45.642 grávidas. Apenas um dos estudos (que incluiu 490 grávidas e 423 crianças) mostrou associação positiva, estatisticamente significativa. Esta MA não suportou a causalidade entre a suplementação de ácido fólico na grávida e o aumento do risco de desenvolvimento de asma na infância. Pelo reduzido número de estudos incluídos na MA e pela heterogeneidade na metodologia dos estudos incluídos, nomeadamente na suplementação com ácido fólico, foi dada a esta MA um nível de evidência (NE) 2.
Yang e colaboradore ${ }^{20}$ publicaram, em 2014, um artigo que inclui uma MA e um EO. Ambos os estudos tiveram como objetivo perceber a associação entre diferentes doses de ácido fólico suplementadas na gravidez e o risco de desenvolvimento de asma na infância. A MA incluiu cinco estudos coorte, com 14.438 participantes. Nenhum dos estudos incluídos estratificou os resultados por dose total de suplementação de ácido fólico, não se verificando associação entre a suplementação com ácido fólico durante a gravidez e o risco de asma na infância. A esta MA foi atribuído um NE 2, pelo reduzido número de estudos incluídos na MA e pela heterogeneidade na metodologia dos estudos incluídos. O EO desenvolvido por Yang e colaboradores $^{20}$ tratou-se de um estudo caso-controlo com 362 crianças (150 com diagnóstico de asma e 212 sem diagnóstico de asma), cuja dose total de suplementação materna com ácido fólico durante a gravidez foi dividida em quatro categorias: zero (sem suplementação), baixa (dose de suplemen-

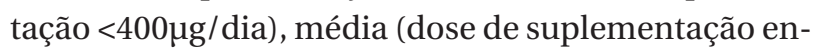
tre 400 e $800 \mu \mathrm{g} / \mathrm{dia}$ ) e alta (dose de suplementação $>800 \mu \mathrm{g} / \mathrm{dia}$ ). No grupo caso foram suplementadas com ácido fólico $76 \%$ das mulheres e no grupo controlo $63,7 \%$, com diferença estatisticamente significativa na análise univariada. A realização de um modelo de regressão logística mostrou associação, estatisticamente significativa, entre a dose de ácido fólico suplementada na gravidez e o risco de desenvolvimento de asma na infância. Assim, verificou-se que a suplementação

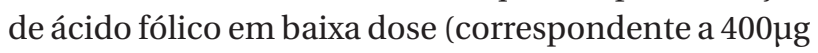
nos primeiros 90 dias de gestação) reduz o risco do desenvolvimento de asma na infância, enquanto a suplementação em altas doses $(800 \mu \mathrm{g}$ em três meses ou 


\begin{tabular}{|c|c|c|c|c|}
\hline Referência & Estudos/População & Metodologia & Resultados & $\begin{array}{l}\text { Nível de } \\
\text { Evidência }\end{array}$ \\
\hline $\begin{array}{l}\text { Crider, et al. } \\
2013^{19}\end{array}$ & $\begin{array}{l}\text { MA } \\
5 \text { estudos ( } 3 \text { coorte } \\
+2 \text { caso controlo) } \\
45.642 \text { grávidas }\end{array}$ & $\begin{array}{l}\text { Suplementação } \\
\text { periconcecional vs } \\
\text { não suplementação } \\
\text { Outcome: } \\
\text { Desenvolvimento de asma } \\
\text { ou pieira na infância }\end{array}$ & $\begin{array}{l}\text { - Apenas um estudo mostrou associação } \\
\text { positiva com } R R=1,06 \\
\text { - Não foi suportada causalidade entre a } \\
\text { suplementação com ácido fólico na } \\
\text { grávida e o risco de asma na infância }\end{array}$ & 2 \\
\hline \multirow[t]{2}{*}{$\begin{array}{l}\text { Yang, et al. } \\
2014^{20}\end{array}$} & $\begin{array}{l}\text { MA } \\
5 \text { estudos coorte } \\
14.438 \text { grávidas }\end{array}$ & $\begin{array}{l}\text { Associação entre } \\
\text { diferentes doses de ácido } \\
\text { fólico suplementadas na } \\
\text { gravidez e o risco de } \\
\text { desenvolvimento de asma } \\
\text { na infância }\end{array}$ & $\begin{array}{l}\text { - Nenhum estudo estratificou os } \\
\text { resultados por dose de ácido fólico } \\
\text { - Não se verificou associação entre a } \\
\text { suplementação com ácido fólico na } \\
\text { gravidez e o risco de asma na infância }\end{array}$ & 2 \\
\hline & $\begin{array}{l}\text { Estudo caso controlo } \\
362 \text { crianças ( } 150 \text { com } \\
\text { diagnóstico de asma e } \\
212 \text { sem diagnóstico de } \\
\text { asma }\end{array}$ & $\begin{array}{l}\text { Outcome: } \\
\text { Desenvolvimento de } \\
\text { asma na infância }\end{array}$ & $\begin{array}{l}\text { - Suplementação de ácido fólico em baixa } \\
\text { dose reduz o risco do desenvolvimento de } \\
\text { asma na infância (OR=0,36, } \\
\text { IC } 95 \%=0,17-0,77) \\
\text { - Suplementação em altas doses } \\
\text { está associada ao aumento do risco de } \\
\text { desenvolvimento de asma na infância } \\
\text { (OR=3,16, IC } 95 \%=1,15-8,71)\end{array}$ & 2 \\
\hline $\begin{array}{l}\text { Woude, et al. } \\
2014^{16}\end{array}$ & $\begin{array}{l}\text { Estudo coorte } \\
\text { retrospetivo } \\
39.602 \text { grávidas } \\
40.628 \text { crianças }\end{array}$ & $\begin{array}{l}\text { Avaliação da incidência } \\
\text { entre a dispensa de } \\
\text { medicação para a asma } \\
\text { para crianças cujas mães } \\
\text { foram suplementadas } \\
\text { com altas doses de ácido } \\
\text { fólico durante a gravidez } \\
\text { Outcome: } \\
\text { Dispensa de medicamentos } \\
\text { para a asma em crianças }\end{array}$ & $\begin{array}{l}\text { - Associação positiva entre a suplementação } \\
\text { com ácido fólico em altas doses e a } \\
\text { dispensa de medicação para a asma } \\
(\mathrm{IRR}=1,26, \mathrm{IC} 95 \%=1,07-1,47)\end{array}$ & 2 \\
\hline $\begin{array}{l}\text { Veeranki, } \\
\text { et al. } 2015^{21}\end{array}$ & $\begin{array}{l}\text { Estudo coorte } \\
\text { retrospetivo } \\
104.428 \text { mães-crianças }\end{array}$ & $\begin{array}{l}\text { Associação entre a o } \\
\text { período de suplementação } \\
\text { com ácido fólico na } \\
\text { gravidez e o } \\
\text { desenvolvimento de asma } \\
\text { na infância } \\
\text { Outcome: } \\
\text { Desenvolvimento de } \\
\text { asma na infância }\end{array}$ & $\begin{array}{l}\text { - Verificou-se um aumento do risco de } \\
\text { desenvolvimento de asma em crianças } \\
\text { cujas mães foram suplementadas com } \\
\text { ácido fólico (quer apenas o primeiro } \\
\text { trimestre, quer para além do primeiro } \\
\text { trimestre) em comparação com as crianças } \\
\text { cujas mães não foram suplementadas com } \\
\text { ácido fólico durante a gravidez } \\
\text { (OR=1,2, IC } 95 \%=1,1-1,3 \text { ) }\end{array}$ & 2 \\
\hline
\end{tabular}


$400 \mu \mathrm{g}$ em seis meses) está associada ao aumento do risco de desenvolvimento de asma na infância. Por se tratar de um estudo caso-controlo foi atribuído NE 2.

Woude e colaboradores ${ }^{16}$ publicaram, também em 2014, um estudo coorte retrospetivo que utilizou a base de dados IADB.nl com 39.602 mulheres grávidas ligadas a 40.628 crianças. Esta base de dados contém o histórico da prescrição farmacológica de grávida à qual está ligada a(s) criança(s) respetiva(s). Foram colhidos os dados entre 1994 e 2011. Este estudo tinha como objetivo avaliar a incidência da dispensa de medicação para a asma para crianças cujas mães foram suplementadas com altas doses de ácido fólico durante a gravidez. Foi definida como alta dose de ácido fólico a suplementação com $5 \mathrm{mg}$ de ácido fólico durante qualquer período da gestação. O estudo verificou uma associação positiva entre a suplementação de ácido fólico em altas doses e a dispensa de medicação para a asma, que aumentou em $26 \%$ a dispensa recorrente de corticoides inalados. A este estudo foi atribuído um nível de evidência 2 por se tratar de um estudo coorte.

Veeranki e colaboradores ${ }^{21}$ publicaram em 2015 um estudo coorte retrospetivo com 104.428 mães (e respetivos filhos), com crianças nascidas entre 1996 e 2005. Este estudo tinha como objetivo investigar a associação entre a suplementação com ácido fólico na gravidez $\mathrm{e}$ o desenvolvimento de asma na infância. Neste estudo, os resultados não foram estratificados por dose total de ácido fólico, mas por período de suplementação de ácido fólico (sem suplementação, suplementação apenas no $1^{\circ}$ trimestre, suplementação após o $1^{\circ}$ trimestre ou suplementação para além do $1^{\circ}$ trimestre). Não há também referência às doses de ácido fólico utilizadas em cada período. Verificou-se um aumento do risco de desenvolvimento de asma em crianças cujas mães foram suplementadas com ácido fólico (quer apenas o primeiro trimestre, quer para além do primeiro trimestre) em comparação com as crianças cujas mães não foram suplementadas com ácido fólico durante a gravidez. A este estudo foi atribuído NE 2 por se tratar de um estudo coorte.

\section{CONCLUSÕES}

A evidência atual para a associação entre a suplementação com ácido fólico durante a gravidez e o risco de desenvolvimento de asma durante a infância é controversa. No entanto, nos estudos cujos resultados foram estratificados por dose de suplementação com ácido fólico foi consensual que a alta dose (>800 $\mu$ g) de suplementação com ácido fólico estava associada ao aumento do risco de desenvolvimento de asma na infância (SORT B).

De notar que os estudos que não mostram esta associação tratam-se de estudos cuja dose da suplementação não é tida em conta e cuja suplementação ocorreu no período preconcecional e primeiro trimestre (concordantes com as recomendações existentes), não tendo ocorrido suplementação a partir do segundo trimestre.

No estudo caso-controlo desenvolvido por Yang e colaboradores, ${ }^{20}$ quando realizada a análise dos resultados sem ter em contra a estratificação dos mesmos por dose de ácido fólico suplementada, não se verifica relação estatisticamente significativa entre a suplementação com ácido fólico na gravidez e o desenvolvimento de asma na infância. Apenas após a análise estratificada dos resultados se verificou esta associação.

O limitado número de estudos incluídos nesta revisão, bem como a heterogeneidade das metodologias dos mesmos, são as principais limitações identificadas pelos autores desta revisão.

Na perspetiva dos autores, esta revisão pretende não só alertar para uma possível associação, ainda pouco debatida, entre a suplementação com altas doses de ácido fólico durante a gravidez e o desenvolvimento de asma na infância, mas fundamentalmente tem como objetivo sensibilizar todos os profissionais para a prescrição mais adequada de ácido fólico na gravidez. Efetivamente, estima-se que a suplementação com $5 \mathrm{mg}$ de ácido fólico seja frequente, tendo em conta as formulações existentes no país. Assim, os autores entendem como importante, eventualmente uma medida de prevenção quaternária, a maior sensibilização para a prescrição de ácido fólico na dose de $400 \mu \mathrm{g}$ em todas as gestantes sem indicação para a suplementação com $5 \mathrm{mg}$ de ácido fólico.

Futuramente serão necessários estudos de maiores dimensões, com metodologia mais homogénea, de modo a estabelecer com maior força a associação entre a suplementação com altas doses de ácido fólico durante a gravidez e o risco de desenvolvimento de asma na infância. 


\section{REFERÊNCIAS BIBLIOGRÁFICAS}

1. Lumley J, Watson L, Watson M, Bower C. Periconceptional supplementation with folate and/or multivitamins for preventing neural tube defects. Cochrane Database Syst Rev. 2001;(3):CD001056.

2. Blencowe H, Cousens S, Modell B, Lawn J. Folic acid to reduce neonatal mortality from neural tube disorders. Int J Epidemiol. 2010;39 Suppl 1:i110-21.

3. Wald NJ. Folic acid and the prevention of neural-tube defects. N Engl J Med. 2004;350(2):101-3.

4. MRCVitamin Study Research Group. Prevention of neural tube defects: results of the Medical Research Council Vitamin Study. Lancet. 1991;338(8760):131-7.

5. Organização Mundial da Saúde. Diretriz: suplementação diária de ferro e ácido fólico em gestantes [Internet]. Genebra: OMS; 2013. Available from: http://apps.who.int/iris/bitstream/10665/77770/9/ 9789248501999_por.pdf

6. Direção-Geral da Saúde. Programa nacional para a vigilância da gravidez de baixo risco. Lisboa: DGS; 2015. ISBN 9789726752332

7. Jong-Potjer LC, Beentjes M, Bogchelman M, Jaspar AHJ, Van Asselt KM. NHG-Standaard Preconceptiezorg [NHG Standard 'Preconception care']. Huisarts Wet. 2011;54(6):310-26. Dutch

8. Peña-Rosas JP, Viteri FE. Effects and safety of preventive oral iron or iron+folic acid supplementation for women during pregnancy. Cochrane Database Syst Rev. 2009;(4):CD004736.

9. Wilson RD, Désilets V, Wyatt P, Langlois S, Gagnon A, Allen V, et al. Preconceptional vitamin/folic acid supplementation 2007: the use of folic acid in combination with a multivitamin supplement for the prevention of neural tube defects and other congenital anomalies. J Obstet Gynaecol Can. 2007;29(12):1003-1013.

10. Asher MI, Keil U, Anderson HR, Beasley R, Crane J, Martinez F, et al. International study of asthma and allergies in childhood (ISAAC): rationale and methods. Eur Respir J. 1995;8:483-91.

11. Håberg SE, London SJ, Stigum H, Nafstad P, Nystad W. Folic acid supplements in pregnancy and early childhood respiratory health. Arch Dis Child. 2009;94(3):180-4.

12. Whitrow MJ, Moore VM, Rumbold AR, Davies MJ. Effect of supplemental folic acid in pregnancy on childhood asthma: a prospective birth cohort study. Am J Epidemiol. 2009;170(12):1486-93.

13. Troen AM, Mitchell B, Sorensen B, Wener MH, Johnston A, Wood B, et al. Unmetabolized folic acid in plasma is associated with reduced natural killer cell cytotoxicity among postmenopausal women. J Nutr. 2006;136(1):189-94.

14. Waterland RA, Michels KB. Epigenetic epidemiology of the developmental origins hypothesis. Annu Rev Nutr. 2007;27:363-88.

15. Hollingsworth JW, Maruoka S, Boon K, Garantziotis S, Li Z, Tomfohr J, et al. In utero supplementation with methyl donors enhances allergic airway disease in mice. J Clin Invest. 2008;118(10):3462-9.

16. Zetstra-van der Woude PA, De Walle HE, Hoek A, Bos HJ, Boezen HM, Koppelman $\mathrm{GH}$, et al. Maternal high-dose folic acid during pregnancy and asthma medication in the offspring. Pharmacoepidemiol Drug Saf. 2014;23(10):1059-65.

17. INFARMED. Prontuário terapeutico on-line [homepage]. Lisboa: Autoridade Nacional do Medicamento e Produtos de Saúde; 2016 [cited 2016 Aug 14]. Available from: http://app10.infarmed.pt/prontuario/index.php

18. Ebell MH, Siwek J, Weiss BD, Woolf SH, Susman J, Ewigman B, et al. Strength of recommendation taxonomy (SORT): a patient-centered approach to grading evidence in the medical literature. Am Fam Physician. 2004;69(3):548-56.

19. Crider KS, Cordero AM, Qi YP, Mulinare J, Dowling NF, Berry RJ. Prenatal folic acid and risk of asthma in children: a systematic review and meta-analysis. Am J Clin Nutr. 2013;98(5):1272-81.

20. Yang L, Jiang L, Bi M, Jia X, Wang Y, He C, et al. High dose of maternal folic acid supplementation is associated to infant asthma. Food Chem Toxicol. 2015;75:88-93.

21. Veeranki SP, Gebretsadik T, Mitchel EF, Tylavsky FA, Hartert TV, Cooper WO, et al. Maternal folic acid supplementation during pregnancy and early childhood asthma. Epidemiology. 2015;26(6):934-41.

\section{CONFLITO DE INTERESSES}

Os autores declaram não ter quaisquer conflitos de interesse.

\section{ENDEREÇO PARA CORRESPONDÊNCIA}

Andreia Ramôa

E-mail: andreia_ram@hotmail.com

Recebido em 09-09-2016

Aceite para publicação em 05-02-2018 


\section{ABSTRACT \\ MATERNAL HIGH-DOSE FOLIC ACID DURING PREGNANCY AND RISK OF ASTHMA IN CHILDREN: AN EVIDENCE BASED REVIEW}

Objective: Maternal supplementation with folic acid has a positive effect on the prevention of neural tube defects. The relationship between high-dose supplementation of folic acid (5mg) and the development of asthma in children is controversial. The aim of this review is to appraise the evidence on the association between the supplementation with high doses of folic acid during pregnancy and the risk of developing asthma in childhood.

Data sources: National Guideline Clearinghouse, Canadian Medical Association Practice Guidelines, Cochrane Library, DARE, Bandolier, PubMed and Index of Portuguese Medical Journals.

Review method: We searched meta-analyses, systematic reviews, original studies and clinical guidelines published between July/2006 and July/2016 in Portuguese, English, Spanish and French languages, using the MeSH terms: 'Folic Acid', 'Asthma' and 'Pregnancy'. The Strength of Recommendation Taxonomy (SORT) scale of the American of Family Physicians was used to assign evidence levels and strength of recommendation grades.

Results: Five studies were selected from a total of 29 articles found, including two meta-analyses and three original studies. Results from meta-analyses do not support an association between periconceptional folic acid supplementation and increased risk of developing childhood asthma. However, none of the studies included in the meta-analyses stratified the results according to the dose of folic acid used during pregnancy and risk of asthma. On the other hand, original studies suggest that supplementation with high doses of folic acid during pregnancy may increase the risk of developing asthma in childhood.

Conclusions: Evidence on the association between folic acid supplementation during pregnancy and the risk of developing asthma during childhood is controversial. Studies showing results stratified according to the dose of folic acid reveal that high doses (>800 $\mathrm{\mu g}$ ) are associated with an increased risk of developing childhood asthma (SORT B).

Keywords: Folic acid; Asthma; Pregnancy. 